\title{
LOS SENTIDOS Y SIGNIFICADOS DE LAS
} PLATAFORMAS DE CITAS

Valeria Correa Santa*, Luisa Fernanda Guerrero Herrera**, David Gutiérrez Hernández***

\section{Resumen}

El objetivo del proyecto es analizar la creación de sentidos y significados que tienen las personas frente a las relaciones interpersonales; determinar cómo influyen las habilidades sociales y las múltiples experiencias previas para llegar al uso de una plataforma de citas; comprender si las relaciones en los adolescentes se modifican después de haber interactuado con la plataforma de citas, teniendo en cuenta que el sentido que se da con el paso del tiempo y en ámbitos culturales o sociales, la sociedad se ve influenciada por las redes.

Los procesos cognitivos son intermediarios para configurar nuevas formas de entender las relaciones afectivas; es por esto por lo que el rol de la interacción entre ambas categorías es esencial. Las redes sociales modificaron las formas de comunicación y con las nuevas tecnologías, las formas de amar. Las personas que se conocen a través de una plataforma de citas y establecen vínculos efímeros y líquidos.

* Estudiante de Psicología, VI semestre de la Fundación Universitaria del Área Andina, Semillero de investigación Acedai. Correo: vcorrea5@estudiantes.areandina.edu.co

* Estudiante de Psicología, VI semestre de la Fundación Universitaria del Área Andina, Semillero de investigación Acedai. Correo: Iguerrero36@estudiantes.areandina.edu.co

*** Docente del programa de psicología de la Fundación Universitaria del Área Andina, Grupo Paideia. Correo: 


\section{Introducción}

El objetivo de esta investigación es conocer los sentidos y significados que construyen los universitarios con base en las habilidades sociales y la influencia en la creación de relaciones interpersonales, las cuales se generan mediante la interacción del uso de las plataformas de citas, teniendo en cuenta las categorías fundamentales de Vygotsky (1934) de sentido. Los conocimientos existentes abarcan que las habilidades sociales inciden como experiencias previas para recurrir a las redes sociales y, a su vez, afirman que prefieren el contacto virtual para ser más auténticos. Esta investigación es de tipo cualitativo, porque permite conocer las cualidades de las personas y ver cómo se construyen las categorías psicológicas a partir de la interacción con las plataformas de citas.

Las redes sociales en la actualidad cumplen una función muy importante para los individuos, porque influyen en las actividades diarias y en la manera de crear relaciones interpersonales.

Además, se muestra la relación entre la adicción a las redes sociales y las habilidades sociales, enfatizando en la forma de comunicación que se emplea en el mundo actual por parte de los adolescentes a través del Internet y analizando las dimensiones de las habilidades sociales del mismo adolescente con su entorno social (Domínguez e Ybáñez, 2016, p. 182).

Choliz y Marco (2012) consideran que las redes sociales son las herramientas más utilizadas por jóvenes y adolescentes, ya que tienen la posibilidad de am- pliar el círculo de amigos, así como de conectarse de manera sencilla con otras personas para compartir experiencias o información (Domínguez e Ybáñez, 2016, p. 185).

El uso frecuente de las redes sociales se convierte en una necesidad para realizar las actividades de placer, por eso constituyen una de las principales fuentes de ocio para los/as adolescentes, verdaderos/as nativos/as digitales, que han encontrado en estos medios en donde compartir información, establecer relaciones y comunicarse; incluso, en ocasiones pueden preferir este tipo de medios al cara a cara en las conversaciones (Alonso et al., 2015, p. 1).

De esta manera, las redes sociales son un medio para crear espacios donde las personas buscan y pueden lograr una relación interpersonal. Se busca obtener aceptación, puesto que la información que se brinda depende de los datos que la persona decida expresar en su perfil.

En otro caso, las aplicaciones de citas sirven como medio para que las personas conozcan parejas sexuales o establezcan una relación amorosa. En estas aplicaciones, las personas buscan características físicas muy claras acorde con sus preferencias. Cada persona pone en su perfil fotos y descripciones de sus gustos y demás características, llamando la atención de otros sujetos. Las plataformas de citas en "las redes sociales y las nuevas tecnologías modificaron las formas de comunicar y de amar. Las personas se conocen a través de una pantalla y establecen vínculos desde allí: vínculos efímeros, líquidos, superfluos" (Bonavita, 2010, p. 2). 
Las plataformas de citas permiten conocer personas nuevas con diferentes personalidades e incluso, según Turkle (1997), también mostrarse auténtico, puesto que la interacción se realiza a través de una pantalla y el cara a cara pasa a un segundo plano. Una de las paradojas de estas modificaciones de la tecnología son las relaciones virtuales, que permiten al usuario coincidir con personas que tienen intereses en común. Las relaciones que se construyen en la virtualidad permiten quitarse el antifaz y mostrar su "yo real". Por esto, las personas que son tímidas y no se desenvuelven con facilidad, deciden declarar los sentimientos a sus parejas, y algunos adultos a dejar ver sus aspectos de comportamiento reprimidos.

Por otra parte, estas aplicaciones facilitan las interacciones sin importar que sea social, sexual o amorosa. También tienen un papel negativo, dado que algunas de las personas que usan estas aplicaciones no tienen una habilidad social competitiva y esto hace que presenten problemas con las redes sociales: un sujeto con una personalidad vulnerable, con una cohesión familiar débil y con unas relaciones sociales pobres corre un gran riesgo de hacerse adicto si cuenta con un hábito de recompensas inmediatas, tiene el objeto de la adicción a mano, se siente presionado por el grupo y está sometido a circunstancias de estrés (fracaso escolar, frustraciones afectivas o competitividad) o de vacío existencial (aislamiento social o falta de objetivos) (Echeburúa y Corral, 2010, p. 94).

Debido al contexto que rodea el proyecto de investigación, surgió la pregunta de investigación de comprender los sentidos y significados que los adolescentes construyen frente a las relaciones interpersonales, con base en el uso de las plataformas de citas. Vygotsky (1934) propone en su teoría sociocultural, que el aprendizaje de las personas se genera mediante una interacción con la sociedad. Además, el sentido y el significado que Vygotsky le da al uso de la palabra es fundamental, porque en el primer momento plantea que el lenguaje es una herramienta primordial en el niño y a medida que este crece se desarrolla independientemente al pensamiento; sin embargo, ambos cambian y evolucionan. Tanto los procesos psicológicos como el significado que se crean inmersos en la sociedad, muestran la realidad en la que evoluciona y así se van forjando. Además, la conexión entre dichas categorías resulta ser fundamental para el desarrollo de los individuos.

De acuerdo con Bauman (2003), el aporte de cómo la sociedad es transitoria y a partir de esta característica se enmarcan las relaciones, puesto que la modernidad globaliza el amor flotante y da una metáfora de la sociedad líquida. Se plantea que a medida que pasa el tiempo se da una transformación y flexibilidad constante que modifica la forma en que se crean los lazos y el miedo a establecer relaciones duraderas.

Byung-Chul Han (2014): su contribución es con su metáfora del enjambre digital, en la cual plantea que las personas son aisladas y carentes de alma y espíritu. Estos individuos no son capaces de crear un 'nosotros' y esto hace que no se manifiesten como una voz sino como un 
ruido; y lo que caracteriza a la actualidad es la soledad y no la multitud.

Identificar los sentidos y significados que construyen los universitarios, descubriendo las motivaciones, intereses, experiencias actuales y pasadas que tienen los adolescentes para recurrir a las aplicaciones de citas. Su uso les permite relacionarse con las otras personas y, a partir de esa interacción, empezar a construir relaciones interpersonales. Conociendo la frecuencia, los adolescentes usan las plataformas de citas para interactuar con otras personas según los gustos y deseos que tengan.

Así se logra examinar el sentido y significado sobre las relaciones interpersonales, sean duraderas o efímeras, que se han establecido a partir del uso de las plataformas de citas en adolescentes universitarios entre los 18 y 21 años de la Fundación Universitaria del Área Andina de Pereira.

\section{Metodología}

En esta investigación se utilizará la metodología cualitativa, puesto que permite la observación y la recolección de datos no numéricos. También se buscan conocer las cualidades de las personas, lo cual convierte al método cualitativo en el más apropiado para comprender los sentidos y significados, siendo estas categorías psicológicas que se construyen a partir del uso de las aplicaciones de citas. Además, permitirá entender algunos aspectos que tienen los adolescentes en la construcción de las relaciones afectivas mediante las interacciones con las personas en las plataformas.

174 Para Sampieri et al. (2014): "La investigación cualitativa se fundamenta en una perspectiva interpretativa centrada en el entendimiento del significado de las acciones de seres vivos, sobre todo de los humanos y sus instituciones (busca interpretar lo que va captando activamente)" (p. 9).

Sampieri, en su libro Metodología de la investigación, muestra que la investigación cualitativa se enfoca:

En comprender los fenómenos, explotándolos desde la perspectiva de los participantes en un ambiente natural y en relación con su contexto. Además permite entender algunos aspectos que tienen los adolescentes en la construcción de las relaciones afectivas a través de las interacciones con las personas en las plataformas. El enfoque cualitativo se selecciona cuando el propósito es examinar la forma en que los individuos perciben y experimentan los fenómenos que los rodean, profundizando en sus puntos de vista, interpretaciones y significados. (Sampieri et al., 2014, p. 358)

La población de estudio serán los estudiantes de la Fundación Universitaria del Área Andina de Pereira con edades entre los 18 y 21 años. Al ser adolescentes, se constituyen en su gran mayoría como verdaderos nativos digitales que podrían frecuentar estas plataformas de citas.

El enfoque será fenomenológico por la necesidad de comprender los fenómenos en torno al uso de la plataforma y establecer las relaciones afectivas que se generan mediante las aplicaciones de citas.

"Su propósito principal es explorar, describir y comprender las experiencias de las personas con respecto a un fenómeno y descubrir los elementos en común de tales 
vivencias" (Sampieri et al., 2014, p. 493). Las herramientas que se utilizarán son los grupos focales, encuestas y entrevistas que permitan profundizar sobre las experiencias y su relación con la aplicación.

\section{Discusión}

En los resultados esperados de esta investigación, se espera identificar las motivaciones, experiencias e intereses que tienen los adolescentes al momento de usar las plataformas de citas y su frecuencia considerando sus deseos, necesidades, emociones y gustos. También, reconocer las formas de interacción que tienen los sujetos con la aplicación y cómo utilizan esta al momento de relacionarse con otras personas de su interés mediante el uso de las plataformas de citas.

Por último, entender las relaciones interpersonales que se construyen en la modernidad, ya sean duraderas o efímeras, en especial aquellas surgidas a partir del uso de plataformas de citas.

\section{Impactos}

En la actualidad es importante entender las nuevas formas de construcción de los sentidos y significados, que surgen con base en la interacción de las nuevas tecnologías. Por tal motivo, esta investigación tiene impactos a nivel cultural, ya que se pretenden mostrar algunas formas de construcción de procesos psicológicos rudimentarios ligados a las formas de participación e interacción que se puedan construir con las aplicaciones de citas, en este caso las relaciones afectivas.

Es importante notar que el uso adecuado permite buenas interacciones y crea la al- fabetización digital, que consiste en conceptos de la actualidad que se manejan entre las personas que usan la plataforma. Entender que a veces las plataformas tienen un sentido y que puede variar el significado según el uso que se le da.

\section{Referencias}

1. Alonso, P., Rodríguez, Y., Lameiras, M., \& Carrera, V. (2015). Hábitos de uso en las redes sociales de los y las adolescentes: análisis de género. Revista de Estudios e Investigación en Psicología y Educación, vol. extr., 13.

2. Bauman, Z. (2008). Modernidad líquida y fragilidad humana. Nómadas. Critical Journal of Social and Juridical Sciences, 19(3), 1-9.

3. Bonnavita, P. (2015). El amor y los tiempos de Tinder. Cultura y Representaciones Sociales, 10(19), 197-210.

4. Chóliz, M., \& Marcos, M. (2012). Adicción a las redes sociales y habilidades sociales en estudiantes de una institución educativa privada. Propósitos y Representaciones, 4(2), 181-230.

5. Domínguez, J., \& Ibáñez, J. (2016). Adicción a las redes sociales y habilidades sociales en estudiantes de una institución educativa privada. Propósitos y Representaciones, 4(2), 181-205.

6. Echeburúa, E., \& Corral, P. (2010). Adicción a las nuevas tecnologías y a las redes sociales en jóvenes: un nuevo reto. Adicciones, 22(2), 91-96.

Byung-Chul Han. (2014). En el enjambre. Barcelona, España.

8. Sampieri, R., Fernández, C., \& Baptista. P. (2014). Metodología de la investigación. Interamericana Editores, 2(736), 1-634.

7. Vygotsky, L. (1934). Pensamiento y lenguaje. Obras Escogidas, tomo II. Taringa. 